\title{
Review of Contact Urticaria Syndrome-Evaluation to Treatment
}

Marcella Aquino, $M D^{1,2, *}$

Stephanie Mawhirt, $D 0^{1,3}$ Luz Fonacier, $M D^{1,2}$

\author{
Address \\ ${ }^{1}$ SUNY, Stony Brook, USA \\ ${ }^{*}, 2$ Winthrop Rheumatology, Allergy and Immunology, 120 Mineola Blvd., Suite \\ 410, Mineola, NY, 11501, USA \\ Email: maquino@winthrop.org \\ ${ }^{3}$ Winthrop Department of Medicine, 222 Station Plaza North, Suite 509, Mineola, \\ NY, 11501, USA \\ Email:smawhirt@winthrop.org \\ Email: Ifonacier@winthrop.org \\ Published online: 17 0ctober 2015 \\ (C) Springer International Publishing AG 2015
}

This article is part of the Topical Collection on Contact Dermatitis

Keywords Contact urticaria (CoU) • Contact urticaria syndrome (CoUS) - Immunologic contact urticaria (ICoU) -

Non-immunologic contact urticaria (NICoU)

\section{Background}

Contact urticaria (CoU) and contact urticaria syndrome (CoUS) were defined in the 1970 s by Fisher and Maibach and Maibach and Johnson, respectively. CoU was described as the development of a wheal and flare reaction of the skin or mucosa where an external agent contacts the skin [1]. Symptoms typically occur within 20-30 min of exposure and will dissipate within $24 \mathrm{~h}$ [2]. Subsequently, Maibach and Johnson [3] classified CoUS into four stages. Stage 1 involves a localized reaction with erythema and swelling and symptoms of burning, tingling, or itch. Stage 2 consists of a generalized cutaneous reaction with wheal and flare response. Stage 3 involves extracutaneous symptoms including rhinitis, asthma, conjunctivitis, gastrointestinal symptoms, and/or orolaryngeal swelling, and stage 4 consists of anaphylactic shock.

The incidence of CoU in the general population is unknown although certain professions including health care workers (dental, medical, veterinary), agricultural workers, florists/gardeners, food handlers/preparers, hairdressers, and maintenance crews (painters, electricians, welders, and plumbers) carry a higher risk of developing CoU [4-6]. Other at-risk occupations include seafood handlers (fisherman) and laboratory technicians $[7,8]$.

CoU in health care workers in Europe is estimated to be between 5 and $10 \%$ [6]. A 12-year retrospective survey [5] in Australia found that the most common sites for CoU included the hands, arms, and face; the most common allergen was latex (52\%) and most patients were female $(63 \%)$.

A survey [4] conducted to members of the American Contact Dermatitis Society in 2010 sought to clarify how often its members were assessing for $\mathrm{CoU}$ via skin testing and how frequently they encountered CoU in their practices. Of 131 respondents, $1 \%$ felt that CoU was "extremely common," 
$11 \%$ felt it was "common," 39 \% felt it was "infrequent," $32 \%$ felt it was "rare," and $17 \%$ felt it was "extremely rare." Skin prick testing or scratch testing was performed by only $19.1 \%$ of member physicians with one quarter only performing testing for latex. The identification and management of CoU potentially represent a scope of practice gap for dermatologists and allergists.

\section{Pathophysiology}

CoU is classified into two main groups based upon pathophysiologic mechanisms: immunologic and non-immunologic. Non-immunologic contact urticaria is the more prevalent type of contact urticaria $[6,9$, 10]. Additionally, there are a few identified contact urticants that seem to share features consistent with an overlap of both immunologic and non-immunologic mechanisms. Both types of CoU involve percutaneous absorption of the exogenous urticant antigen (urticariogen) into the epidermis and/or dermis depending on skin integrity or breakdown [10]. Further biochemical research is certainly required to definitively illustrate the immunologic signaling and cellular mechanisms activated by contact urticants.

Immunologic contact urticaria (ICoU) is an allergic type I IgE-mediated hypersensitivity reaction in which the patient has been previously sensitized to a contact urticant. Prior exposure via mucocutaneous, respiratory, or gastrointestinal routes leads to allergic sensitization via the production of allergen-specific IgE, which in turn precipitates an immediate hypersensitivity reaction upon allergen re-exposure $[7,9,10]$. Substances with molecular weights greater than 10,000 (proteins) may cause sensitization themselves, while urticants of molecular weight less than 1000 (chemicals) function as haptens and require a serum carrier protein for sensitization to occur [11]. Definitive evidence of a type I hypersensitivity reaction may be obtained for some contact urticants through commercially available in vitro assays which detect urticantspecific IgE in patient serum [10].

As a type I hypersensitivity allergic reaction, ICoU maintains the potential to present as a clinical CoUS since histamine is the chief inflammatory mediator [10]. Through mast cell degranulation and histamine release, a spectrum of local to systemic effects may precipitate. Other immune cells and chemical mediators of inflammation that contribute to the allergic reaction of ICoU include basophils, eosinophils, Langerhans cells, leukotrienes, prostaglandins, and kinins [9, 10, 12].

It is theorized that the contact antigens that generate ICoU and CoUS are either more readily absorbed by the skin due to their chemical properties or incite a stronger allergic response compared to non-immunologic agents [10]. Furthermore, multiple repeat exposures to immunologic contact allergens can propagate the CoUS, such that patients are at risk of 
developing reactions beyond stage 1 (localized urticaria), portending higher morbidity and potential mortality [11].

In contrast, non-immunologic contact urticaria (NICoU), as the name implies, is not an allergic hypersensitivity reaction in the classic definition. Since sensitization is not involved, NICoU can develop upon both initial and subsequent exposure to the urticant. NICoU always remains localized to the area of contact, and urticaria severity may be dependent on urticant amount, concentration, and exposure time $[8,10]$. It is presumed that NICoU can develop from the release of epidermal prostaglandins following insult to the local blood vessels. Prostaglandins and leukotrienes are the proposed mediators of NICoU since oral and topical non-steroidal anti-inflammatory drugs provide a clinical treatment response $[2,9,10,13,14]$. Prostaglandin D2 specifically has been isolated as a mediator in NICoU reaction to benzoic acid and sorbic acid [15].

The variable reaction severity of NICoU at different contact sites has been a peculiarity and sparks further interest into the exact mechanism of this CoU type. Greater reactions occur at the skin of the face and back compared to the palms and soles, which may be due to differences in vascularity, permeability, epidermal-dermal thickness, or integumentary microenvironment [10]. The precise mechanisms that induce NICoU are varied based on the culprit substance. Some urticants directly damage local capillaries leading to non-IgEmediated histamine release from mast cells, or vasoactive amine, leukotriene, prostaglandin, and acetylcholine release $[6,8]$.

\section{Contact urticaria of unclear mechanism}

There is an additional type of CoU which exhibits shared features of both immunologic and non-immunologic mechanisms. A definitive pathophysiology of these reactions has not yet been completely clarified. A well-known example of this type of overlap CoU is the oxidizing chemical ammonium persulfate (contained in hair bleaching products) [12]. Not only is type I hypersensitivity involved in the pathogenesis of ammonium persulfate-induced CoU as specific IgE may be detected in patient serum, but the classical complement pathway is also activated by IgM and IgG.

Increased reporting on the multitude of substances that elicit CoU has led to an overall heightened awareness of this disorder among the medical community over the last 40 years. Still fundamental to the diagnosis of CoU is a comprehensive history and physical examination which guides appropriate confirmatory diagnostic testing, utilizing the suspected contact allergens.

Key history regarding the details surrounding the potential offending contact urticant as well the symptomatology and clinical signs experienced by the patient should be obtained. The CoU and CoUS must be differentiated from other urticarial disorders, particularly the physical urticarias such as delayed pressure, vibratory, aquagenic, and cholinergic urticarias. Moreover, it should be noted that two or more sub-types of 
urticaria may potentially co-exist, either through similar or differing pathophysiologic mechanisms [16].

Nevertheless, as with all types of urticaria, physicians should obtain the following information regarding the urticarial lesions: onset following substance contact; evolution, duration, and resolution; characteristics including frequency and number of lesions, localized or generalized body distribution, pattern distribution (discrete, punctuate, linear, follicular), size, shape, and confluency; location of erythema and pallor; and angioedema and other associated symptoms such as pruritus, burning, or painful tingling sensations [8]. Moderate to severe pruritus is a key consistent feature, based on many case reports, and is more intense in immunologic variants [17]. Any area of skin may be theoretically affected, although exposed areas such as the hands, extremities, and face are the most frequently reported [9]. The oral mucous membranes and oropharynx may be affected in the oral allergy syndrome, described in a latter section. Inquiry on the presence of non-cutaneous manifestations such as conjunctivitis, rhinitis, respiratory symptoms/asthma, gastrointestinal disturbance, and hemodynamic instability including anaphylaxis is crucial to history gathering.

The patient should also be questioned about any treatments used and their efficacies. Temporal relationship to the time of day, week, or month, season, environment, or hobbies should be obtained. Additionally, patients should be asked about current and new medications, foods, home improvement work, and personal hygiene or cosmetic products. Supplementary history encompasses general allergic disease history, autoimmune disorders, immunosuppressive medications, family history, social history [9] and presence of other nonspecific symptoms (stress), recent travel, relationship to menstrual cycle, and effect of the urticaria on the quality of life [18].

Atopic disease history especially asthma and atopic dermatitis is of importance. Atopy has been determined to be a significant risk factor for the predisposition to develop ICoU and the CoUS [6, 11]. In a 1985 review of CoU, it was reported that $46 \%$ of patients reporting a clinical picture consistent with contact urticaria also reported an atopic history [17]. A positive atopic history is associated with CoU to natural rubber latex products, certain foods, and ammonium persulfate [5]. Atopy has also been associated with a greater sensitization process for protein contact urticants [19].

Obtaining an occupational exposure history from the patient is essential. $\mathrm{CoU}$ was established as the second most common workplace-related disease entity in Finland, according to the "Finnish Register of Occupational Diseases" report from 1990 to 1994 [20]. Furthermore, the previously mentioned retrospective analysis of 151 patients conducted by Williams et al. [5] in Australia demonstrated that $94.7 \%$ of CoU diagnoses were related to occupation. CoU has been described to cyclic acid anhydrides in welders, painters, plumbers, chimneysweeps, packers, electricians, and produce handlers [21, 22]. Gardeners exposed to plants such as common ivy, rosemary, basil, sage, oregano, marjoram, thyme, and peppermint are also at risk for CoU [22-24]. Occupational contact urticants are geographically varied.

\section{Physical exam}

The history obtained from patients may be vague; therefore, the dermatologist or allergist must be astute in the evaluation of CoU [25]. According to a 1992 
review by Harvell et al., the prototypical urticarial reaction presents only in a minority of patients with a diagnosis of CoU [10]. It is suspected that CoU is a missed diagnosis and/or underreported by both patients and physicians in the USA. After obtaining a thorough history, physical examination of the patient is the next step before proceeding to diagnostic laboratory testing. The physical examination may occur during active episodes or quiescence. Here, we will discuss in tandem the patient-reported findings and physical examination in the description of a contact urticarial reaction.

The wheal and flare response of immunologic CoU has a classic hive appearance: a lesion with central swelling, erythema, and warmth, surrounded by a peripheral pallor that is similar to other allergic urticarial disorders [12, 26, 27]. CoU is generally accompanied by pruritus and angioedema at the site of the urticaria. On other hand, NICoU tends to appear as localized erythema with angioedema, but not necessarily as a typical wheal and flare response [10, 17]. Skin affected may be fully intact or urticaria may develop on an irritated, eczematous skin surface [10, 12]. The majority of patients report a visible reaction occurring within minutes to an hour after external agent skin exposure [28]. However, some report local pruritus or burning alone [2, 9]. ICoU tends to develop within 15 to $30 \mathrm{~min}$, while non-immunologic cases can have a delayed presentation from 45 min up to an hour $[6,11]$ (Table 1).

Symptoms in both NICoU and ICoU tend to resolve within 1 to $2 \mathrm{~h}$ of the presentation onset but may last for up to $24 \mathrm{~h}$ in some reported cases [9, 12]. Swelling typically resolves prior to the clearing of erythema. After resolution, the

Table 1. Characteristics of contact-induced skin reactions

\begin{tabular}{|c|c|c|c|c|}
\hline Characteristic & $\begin{array}{l}\text { Contact } \\
\text { urticaria }\end{array}$ & $\begin{array}{l}\text { Contact urticaria } \\
\text { syndrome }\end{array}$ & Protein contact dermatitis & $\begin{array}{l}\text { Allergic } \\
\text { contact } \\
\text { dermatitis }\end{array}$ \\
\hline $\begin{array}{l}\text { Onset of } \\
\text { reaction }\end{array}$ & $\begin{array}{l}\text { Immediate within } \\
\text { minutes }\end{array}$ & Immediate & Immediate & Delayed \\
\hline Type of reaction & Wheal and flare & $\begin{array}{l}\text { Wheal and flare or } \\
\text { eczema }\end{array}$ & $\begin{array}{l}\text { Wheal and flare angioedema, } \\
\text { erythema, vesicles, eczematous }\end{array}$ & Eczematous \\
\hline $\begin{array}{l}\text { Systemic } \\
\text { involvement }\end{array}$ & $\begin{array}{l}\text { Limited to areas } \\
\text { of contact }\end{array}$ & $\begin{array}{l}\text { Generalized urticaria, } \\
\text { rhinoconjunctivitis, } \\
\text { asthma, abdominal } \\
\text { pain/diarrhea, } \\
\text { anaphylaxis }\end{array}$ & & $\begin{array}{l}\text { Generally } \\
\text { none } \\
\text { except for } \\
\text { systemic } \\
\text { contact } \\
\text { dermatitis }\end{array}$ \\
\hline Mechanism & $\begin{array}{l}\text { May be } \\
\text { immunologic } \\
\text { or } \\
\text { non- } \\
\text { immunologic } \\
\text { allergens }\end{array}$ & $\begin{array}{l}\text { Immunologic type I IgE } \\
\text { mediated }\end{array}$ & $\begin{array}{l}\text { Unclear but may involve a type I IgE } \\
\text { mediated, type IV cell mediated, } \\
\text { and/or a delayed reaction due to } \\
\text { IgE bearing Langerhans' cells }\end{array}$ & $\begin{array}{c}\text { Immunologic } \\
\text { type IV cell } \\
\text { mediated }\end{array}$ \\
\hline Triggers & $\begin{array}{l}\text { Foods, } \\
\text { medications, } \\
\text { latex, metals, } \\
\text { occupational } \\
\text { allergens }\end{array}$ & $\begin{array}{l}\text { Foods, medications, } \\
\text { latex, metals, } \\
\text { occupational } \\
\text { allergens }\end{array}$ & $\begin{array}{l}\text { Proteins of either animal or plant } \\
\text { origin. }\end{array}$ & $\begin{array}{l}\text { Hapten } \\
\text { contact } \\
\text { allergens }\end{array}$ \\
\hline
\end{tabular}


skin is clear of any residual signs, without damage or dysfunction [12]. Though, persistent or recurrent CoU can leave marks of hypo- or hyper-pigmentation, as seen in a case of occupational natural rubber latex exposure [6].

In cases of NICoU, the urticaria is always localized to the contact sites. In ICoU, there is a potential for urticaria beyond the contact site and extracutaneous/systemic allergic reaction symptoms due to the underlying pathophysiology. Therefore, necessary components of the examination during an acute episode include vital signs, a complete skin and mucous membrane inspection, as well as examination of the conjunctiva, nasal turbinates, oral cavity, pharynx, and cardiorespiratory and gastrointestinal systems.

The skin should be inspected and palpated for any other abnormal findings, particularly an eczematous dermatitis including atopic, allergic contact dermatitis, or irritant contact dermatitis. It is also important to look for other skin disorders such as xerosis, inflammation, abrasions, scrapes, excoriations, burns, or other physical damage that may disrupt the ability of the protective skin barrier to prevent exogenous substances from becoming transcutaneously introduced through the epidermis [7]. Damaged skin allows contact urticants to more easily penetrate the immune-rich microenvironments of the epidermis and dermis. Insult to skin integrity may help to narrow the suspected urticant, as proteins greater than molecular weight of 1000 cannot penetrate intact skin [17]. It is not infrequent that skin examination is unremarkable if the patient presents during a time of non-exposure.

Diagnosis of immediate skin contact reactions

Testing for immediate skin contact reactions after a thorough history and physical exam is usually performed in a stepwise fashion [9]. (Please refer to Fig. 1.) The initial evaluation is the "open test" performed by using $0.1 \mathrm{~mL}$ of the suspected substance and spreading or gently rubbing it over a $3 \times 3 \mathrm{~cm}$ area of normal-looking skin, usually the ventral forearm, the extensor side of the upper arm, or the upper back. The reaction is measured at 20, 40, and $60 \mathrm{~min}$ to avoid missing positive reactions. Both immunologic and non-immunologic reactions may appear within 15-20 min manifesting as edema, erythema, or tiny intraepidermal spongiotic vesicles typical of acute eczema. Nonimmunologic reactions may be delayed up to $1 \mathrm{~h}$. Although rare, ICoU can also have a delayed onset.

If negative, it may be necessary to apply the suspected substance to skin sites suggested by the patient's history or on slightly affected skin or previously affected skin [25]. In such cases, vesicular reactions may appear within 15$20 \mathrm{~min}$.

If this is negative, an occlusive application (patch or chamber) of the test substance on unaffected skin can be done. If this is still negative, then the patch or chamber can be applied on slightly affected skin or previously affected skin. Some physicians however perform skin prick testing right after a negative open test [9].

Prick testing of suspected allergens can be done for immediate contact reactions. Helaskoski et al. [29] had shown that prick tests can be safely used for diagnosing work-related CoU, asthma, and rhinitis caused by isocyanates, chloramine-T, persulfates, and chlorhexidine. However, one should be cautious of the interpretation and clinical significance of the results. For both prick and 


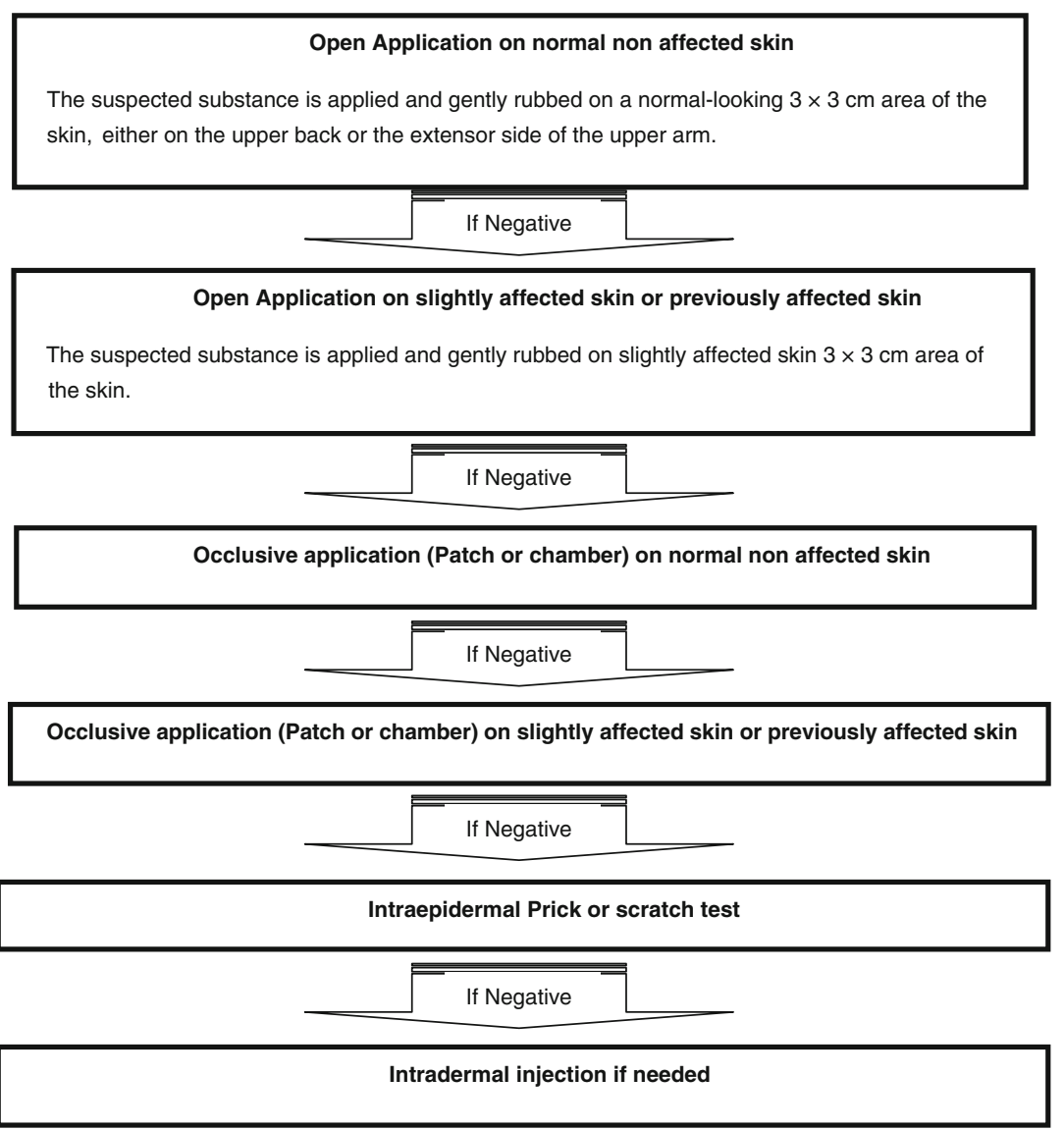

Fig. 1. Diagnosis of immediate skin contact reactions. Adapted from [6].

scratch tests, a positive (histamine hydrochloride) and negative (aqueous sodium hydroxide) control should be performed at the same time. Reading of a prick test is usually performed in $20-30 \mathrm{~min}$.

Although not used as often, the scratch test involves scratching the area with needles (as opposed to pricking the skin with a small amount of allergen with a lancet) after the allergen has been applied. Some authors consider this more useful for non-standardized allergens [9].

When there are extracutaneous manifestations and anaphylaxis symptoms involved, it is important to use serial dilutions to minimize adverse effects. Lifethreatening reactions have been documented during patch testing especially when testing certain occupational substances. Thus, these tests should only be performed by knowledgeable medical personnel and in facilities equipped with resuscitation equipment [30,31]. Intradermal testing has been performed but is rarely needed and carries a greater reaction risk. When testing with poorly or non-standardized substances, control tests should be assessed on at least 20 people to ascertain for false-positive interpretations.

Non-steroidal anti-inflammatory drugs and antihistamines should be avoided prior to the testing as they lead to false-negative results. This is likely based on the lack of response to antihistamines and the positive response to 
acetylsalicylic acid (ASA) and non-steroidal anti-inflammatory drugs (NSAIDs) in NICoU. "It has been proposed that the pathophysiology involves prostaglandin release from the epidermis rather than histamine release from the mast cells, as previously assumed" [13]. In a study by Vernarse et al., in testing NICoU, hydroxyzine and terfenadine "were unsuccessful in inhibiting reactions to benzoic acid, cinnamic acid, cinnamic aldehyde (cosmetics), methyl nicotinate, and dimethyl sulfoxide, suggesting that histamine is not the main mediator" of NICoU [32].

Other in vitro tests have limited availability. Specific IgE to latex is available in the USA, but there is no available standardized skin test. A basophil activation test based on the demonstration of CD 63 expression following exposure of basophils to an allergen (for example, chicken meat) by flow cytometry was reported by Gonzalez-Munoz et al. [33] but is not widely available.

\section{Contact urticaria to foods}

Foods from different classes have been implicated as responsible for contact urticarial lesions including meat (beef, chicken, pork, lamb, turkey), fish (cod, herring), shellfish, vegetables (beans, celery, mushrooms, soy, tomato, cabbage), fruits (apple, mango, orange, peach, strawberry, watermelon), seeds (sunflower, sesame), grains (wheat, flour, rice), and flavorings/spices (cinnamon oil, menthol, vanillin, curry, thyme, paprika) [6]. Other vegetables that have been reported to cause CoU include arugula and parsley [34] which caused lip and tongue angioedema and oropharyngeal pruritus in a 24 -year-old female with positive prick testing to the arugula and parsley leaves.

$\mathrm{CoU}$ to foods is a major cause of occupational-related disease in the food industry [15]. CoU from potatoes can be quite severe and it is a common etiology of food-induced symptoms in chefs [15].

The oral allergy syndrome (OAS) is a commonly encountered mucosal ICoU seen in allergy practices today. It occurs via cross-reactivity between proteins in pollens (birch tree, mugwort weed, ragweed, grass) and in raw fruits (apple, peach, cherry, celery, melon), vegetables (carrot, celery, peppers, potato), nuts, and spices (mustard, sunflower, coriander, aniseed) [15, 35]. OAS is due to a type I immediate hypersensitivity reaction. Patients are sensitized to proteins in pollens (lipid transfer proteins, ribosome inactivating proteins, thaumatin-like proteins, profillins) [35] that share epitopes with the food allergens which will cause degranulation of mast cells and basophils.

Patients experience symptoms that include pruritus; tingling and burning of the lips, palate, oral mucosa, and tongue; throat tightness; and angioedema. Symptoms occur when the raw or unprocessed food is ingested. Typically, the patient can tolerate the cooked food as the protein is denatured from digestive enzymes in the gastrointestinal tract although some foods are not heat sensitive (walnut, pecan, melon, pistachio) or can be heat stable or resistant based on which allergen the patient is allergic to (apple, hazelnut, peanut, celery, tomato) [35]. Symptoms usually occur promptly after exposure to the food allergen. Systemic reactions in cases of oral allergy syndrome have been reported rarely [35]; certain risk factors have been identified including a history of systemic reaction to a food, a positive skin prick test to the commercially prepared allergen, and a prior reaction to peaches or tree nuts [36]. Handling the fruit can also cause CoU [15]. 
Diagnosis of OAS is based on clinical history of allergic rhinitis to pollens, skin prick testing to pollens, skin prick testing to suspected foods using either commercial extracts or prick to prick with raw food, and/or specific IgE to foods. Treatment is limited to the avoidance of the food causing the symptoms.

There are cases in the literature where systemic reactions to foods were preceded by CoU in the patient [37]. Inomota et al. reviewed 15 patients who developed food allergy after epicutaneous sensitization; all but one of these patients were employed as cooks, bakers, or housekeepers [37]. Vegetables were the most common cause in the cooks and housekeepers. The most severe reactions to oral ingestions were seen in sushi cooks who developed anaphylactic reactions after sensitization to fish epicutaneously. Atopic dermatitis and pollen allergy were the other most commonly encountered atopic diseases in this review. In a Spanish study of 19 patients with CoU to peach, 4/19 (21\%) reported that the contact symptoms started prior to the onset of symptoms associated with the ingestion of peach by $2-10$ years [38].

\section{Contact urticaria to medications}

CoU to medications can occur either from the drug itself or from the preservative, bases, or additives used [15]. Medications responsible for ICoU and NICoU include ampicillin/amoxicillin, bacitracin, benzocaine, benzoyl peroxide, capsaicin, cephalosporins, cisplatin, corticosteroids, donepezil, gentamicin, ketoprofen, lidocaine, neomycin, nicotinic acid esters, rifamycin, tar extracts, and uranium salts $[6,15]$.

Systemic and anaphylactic reactions to topical antibiotics including bacitracin have been reported rarely in the literature; around 20 cases of anaphylaxis have been reported [39]. These antibiotics are used in a wide variety of overthe-counter preparations. Bacitracin was among the top five positive patch test results from the 2009-2010 North American Contact Dermatitis Group Data for contact allergens [40]. Case reports include a 54-year-old male [41] with a history of allergic rhinitis and asthma who developed itch, wheezing, hypotension, eyelid edema, and conjunctival injection $5 \mathrm{~min}$ after the application of an antibiotic powder containing bacitracin, neomycin, cysteine, and threonine (Cicatrene ${ }^{\circledR}$ powder). The patient had a positive 20-min reading of patch test to bacitracin. A 22-year-old male [39] developed flushing, generalized itch, lip swelling, difficulty swallowing, tachycardia, and hypotension after applying Neosporin+Pain Relief ${ }^{\circledR}$ (neomycin, polysporin B, bacitracin, and pramoxine). He was skin prick positive to bacitracin prepared in white petrolatum. A 40-year-old female [42] developed facial angioedema, generalized urticaria with itch, nausea, and chest tightness $30 \mathrm{~min}$ after the application of an antibiotic powder containing bacitracin, neomycin, and clioquinol. The patient had positive skin prick testing to both bacitracin and clioquinol.

Systemic symptoms including facial edema, nasal discharge, sneezing, pruritic rash, and mild dyspnea occurred in a 27-year-old female after the application of levofloxacin antibiotic eye drops [43]. 
Topical anesthetics

Chlorhexidine is an underrecognized cause of anaphylaxis from a contact allergen. It is used as an antiseptic and disinfectant and can be found as a coating on medical devices including central lines [44] and urinary catheters. In a retrospective review from the UK of perioperative anaphylaxis [45], chlorhexidine was the responsible agent in $5 \%(n=8)$ of 103 patients where a cause for the anaphylaxis was indentified. Another retrospective review from Europe [46] included 119 patients with suspected perioperative anaphylaxis; an etiology was discovered in 76 patients. Chlorhexidine was the culprit in $5.2 \%$ of cases.

A Danish group [47] sought the best method to assess for chlorhexidine allergy in their cohort of patients. Patients with perioperative anaphylaxis were assessed for chlorhexidine allergy via (1) specific IgE to

chlorhexidine, (2) histamine release assay, (3) skin prick testing (chlorhexidine $5 \mathrm{mg} / \mathrm{ml}$ used), and (4) intradermal testing (chlorhexidine $0.002 \mathrm{mg} / \mathrm{mL}$ ). Twenty-two $(9.6 \%)$ of 228 patients had at least one or more positive tests; all 22 patients had a positive specific IgE, 21 patients had a positive skin prick test, 15 patients had a positive intradermal test, and 12 patients had a positive histamine release assay. Based on these results, they estimated a sensitivity of $100 \%$ and specificity of $97 \%$ for specific IgE and sensitivity of $95 \%$ and specificity of $97 \%$ for skin prick testing to chlorhexidine.

A 55-year-old female developed urticaria [48] after the application of EMLA $^{\circledR}$ cream (lidocaine, prilocaine, castor oil, carboxypolymethylene, and sodium hydroxide); evaluation included an open test, patch testing, and prick and intradermal testing. The open test, patch test to lidocaine, and prick test with EMLA cream were all positive. Another report of CoU to topical anesthetic occurred in a 26-year-old female [49], who developed angioedema of the lips and face 15 min after the application of Pliaglis ${ }^{\circledR}$ (7\% lidocaine, $7 \%$ tetracaine) cream prior to laser treatment for acne. This cream is used as a local anesthetic for various dermatologic procedures [50]. The patient was treated with oral diphenhydramine and her symptoms resolved. A 63-year-old male [51] developed generalized urticaria, itch, and hypotension with loss of consciousness after the application of a topical lidocaine gel (Dynexam ${ }^{\circledR}$ ) by his dentist; he was treated with epinephrine, steroids, and antihistamines and transferred to the hospital. Subsequent evaluation with prick to prick testing was positive to gelling agent (Meyprogat $60^{\circledR}$ ), a component of the lidocaine gel, which is a derivative of guar gum. 
which contained hexylene glycol as the excipient [52]. He was positive via skin prick test and "rub test" to hexylene glycol in 1-10\% concentration.

\section{Contact urticaria to latex}

CoU to latex has long been reported [53], and it is one of the most commonly reported causes of occupational CoU especially in health care workers and other professions. An immunologic IgE [IgE]-mediated mechanism has been identified as well as irritant, non-IgE-mediated mechanism. At risk are populations are patients with spina bifida, contact dermatitis patients, health care workers, food allergic and atopic patients. IgE-mediated immediate-type hypersensitivity is the most common allergic reaction reported by health care workers who use latex medical gloves. Due to preventative measures including latex being largely replaced by other materials, such as neoprene, nitrile, or vinyl [54], a decreased prevalence has been observed [55].

The diagnosis of latex allergy can be challenging. A history of a reaction associated with exposure may be confirmed by tests that include skin testing, serology, or provocation tests. There are 15 International Union of Immunological Societies (IUIS)-documented Hevea latex allergens: Hevea brasiliensis Hev b 1 through Hev b 15. Four Hevea proteins (Hev b 1, 3, 5, and 6.02) [56] are particularly useful as "indicator" allergens for assessment of the allergen content of rubber products or as markers of the presence of latex in the environment. Hev b1 and Hev b3 are the major protein allergens in patients with spina bifida whereas Hev b 5 and Hev b6 are more common in health care workers [15].

In the USA, the commercially available FDA-approved reagent is latexspecific IgE antibody serology with the ImmunoCAP ${ }^{\circledR}$ or Immulite ${ }^{\circledR}$ systems. However, these tests are limited by varying reagent sensitivity and specificity. Skin test reagents have not been approved for use in the USA, and skin testing carries a significant risk for serious reactions. Thus, a negative test in the setting of high clinical suspicion warrants further evaluation and avoidance in these patients. There is also some clinical association between latex allergy and allergic responses to a number of fruits and vegetables, including banana, kiwi, avocado, chestnut, papaya, potato, and tomato. This may be due to Hev b6 whose sequence displays more than $50 \%$ homology to the chitinases in these fruits.

\section{Contact urticaria to allergens causing type IV hypersensitivity reactions}

Miscellaneous metals have been reported to cause immediate-type contact reactions. Most of them are occupational including chromium, cobalt, iridium, nickel, platinum salts, mercury, and rhodium. Immunologic mechanisms have been identified for chromium, cobalt, iridium, nickel, platinum salts, and possibly mercury. IgE antibodies have been demonstrated in sensitized workers reactive to platinum and nickel-serum albumin complexes $[57,58]$. Other metals such as aluminum, copper, gold, 
palladium, ruthenium, tin, and zinc have also been reported to cause CoU, but the mechanism is not yet well defined.

Cosmetics: fragrances, PPD, ammonium persulfate, henna, and other dyes

Cosmetic components can cause CoU with or without systemic symptoms. This may be grossly undiagnosed because patients fail to report these reactions and just discontinue the use of the product.

Fragrances have been reported to cause both immediate and delayed hypersensitivity reactions. A multicenter study in Hungary found that $6.1 \%$ of patients with contact dermatitis to fragrance also report an immediate CoU reaction. The most frequently reported allergens were cinnamic aldehyde, cinnamic alcohol, and Evernia prunastri [59].

Hair dye chemicals such as p-phenylenediamine (PPD) and hair glue are reported causes of CoU. Aside from PPD, reactions to Basic Blue 99 (a mixture of 23-32 substances at various concentrations and with varying compositions), Basic Brown 17 (an azo dye), henna, and other reactive dyes have also been reported to cause CoU [14, 60]. Ammonium persulfate, used for hair bleaching, can also cause NICoU. Poltronieri reports contact urticaria and angioedema after airborne exposure to ammonium persulfate [61]. Hair dressers who are exposed to these products on a daily basis are at risk of developing cutaneous reactions.

\section{Therapy}

The most common therapeutic modalities for contact urticaria include prevention through avoidance and adjunct pharmacological treatments. The main principles of treatment are (1) identification of the contact urticant, (2) elimination of exposure via complete avoidance or preventative contact measures, and (3) symptom control with either conservative therapies or pharmacologic agents.

Following identification of the culprit contact urticant by history and diagnostic testing, patients must be educated on their disorder, understand its potential for evolution over time, and be aware of the therapeutic options available. For ICoU, it is important to stress that recurrent exposures can precipitate the CoUS, with progression to subsequent stages and greater risk of anaphylaxis. It is recommended that patients with ICoU always have injectable epinephrine and antihistamines on hand with them, should they be required to treat a life-threatening reaction [9].

The safest and most effective measure is complete avoidance of the contact urticant [12]. However, complete avoidance in the setting of occupational exposure may not be feasible. In these cases, some patients may obtain adequate prevention with the use of protective skin measures, such as gloves, if practical given the workplace setting. Some types of personal protective 
equipment (PPE) have the potential to exacerbate skin breakdown or eczema through their occlusive effects, which may paradoxically worsen CoU if exposure happens to occur. Other patients may choose daily pharmacologic therapies (described below) as a prophylactic measure, if avoidance or prevention interferes too greatly with their career and/or quality of life.

The UK published standard of care guidelines for occupational contact urticaria in 2013, advocating a multi-disciplinary approach between the patient, physician, and employer in order to ensure occupational health safety [62]. Key recommendations from the British Occupational Health Research Foundation (BOHRF) were to provide workers with PPE, encourage the use of conditioning skin creams or emollients, provide education and training on health safety, and ensure physician assessment when appropriate [62, 63].

\section{Pharmacologic treatments}

First-line medications for symptom relief depend on the CoU type, but the overall goal is to inhibit inflammatory mediator release. The main treatment of ICoU includes the same medications used to treat other chronic urticarias mediated by type I hypersensitivity reactions. Second-generation H1 antihistamines are considered first-line therapy for ICoU as they block the local and systemic effects of histamine. Doses of antihistamines should be increased for maximum symptom control prior to considering alternative therapies [6] as long as no adverse effects are intolerable. Severe cases of CoUS may benefit from a short course of oral steroids for amelioration of symptoms $[6,11,12]$. In cases of NICoU, first-line therapies include aspirin and non-steroidal anti-inflammatory drugs, which act to inhibit the inflammatory effects of prostaglandins and leukotrienes by decreasing their production.

Alternative therapies

The use of other alternative therapies such as ultraviolet light therapy, leukotriene receptor antagonists, or immunomodulators which are used for chronic idiopathic urticaria have not been well described for CoU. There are no standard recommendations on the utilization or consensus on the efficacy of these therapies in CoU. Experimental subcutaneous immunotherapies for select urticants are currently undergoing evaluation. For example, immunotherapy for occupational flour allergy among bakers is being evaluated in Italy [64]. Another Italian group investigated the tolerability of latex sublingual immunotherapy (SLIT) with rush protocol in a double-blinded, placebo-controlled study in patients specifically afflicted by latex contact urticaria. They concluded that the SLIT group tolerated the therapy with no significant adverse reactions and supported the safety of SLIT for the treatment of latex-induced contact urticaria [65].

\section{Conclusion}

CoU is likely an underappreciated disease presenting to dermatologists and allergists. A high index of suspicion is needed to elicit the history that would suggest this disease and then proceed with further evaluation including testing. 
The mainstay of treatment is the identification of the relevant urticant and its avoidance whenever possible. Other therapies that show promise include induction of tolerance via immunotherapy.

\section{Compliance with Ethical Standards}

\section{Conflict of Interest}

Stephanie Mawhirt declares that she has no conflict of interest.

Luz Fonacier declares that she has no conflict of interest.

Marcella Aquino declares that she has no conflict of interest.

Human and Animal Rights and Informed Consent

This article does not contain any studies with human or animal subjects performed by any of the authors.

\section{References and Recommended Reading}

1. Rietschel RL, Fowler JFJ. Contact urticaria. 6th ed. Fisher's contact dermatitis: BC Decker Inc; 2008.

2. Wakelin SH. Contact urticaria. Clin Exp Dermatol. 2001;26(2):132-6.

3. Maibach HI, Johnson HL. Contact urticaria syndrome. Contact urticaria to diethyltoluamide (immediate-type hypersensitivity). Arch Dermatol. 1975;111(6):72630.

4. Orb Q et al. Prevalence and interest in the practice of scratch testing for contact urticaria: a survey of the American Contact Dermatitis Society members. Dermatitis. 2014;25(6):366-9.

5. Williams JD et al. Occupational contact urticaria: Australian data. Br J Dermatol. 2008;159(1):125-31.

6. Gimenez-Arnau A et al. Immediate contact skin reactions, an update of contact urticaria, contact urticaria syndrome and protein contact dermatitis - "a never ending story". Eur J Dermatol. 2010;20(5):552-62.

7. Amaro C, Goossens A. Immunological occupational contact urticaria and contact dermatitis from proteins: a review. Contact Dermatitis. 2008;58(2):67-75.

8. Doutre MS. Occupational contact urticaria and protein contact dermatitis. Eur J Dermatol. 2005;15(6):41924.

9. Bhatia R, Alikhan A, Maibach HI. Contact urticaria: present scenario. Indian J Dermatol. 2009;54(3):264-8.

10. Harvell J, Bason M, Maibach HI. Contact urticaria (immediate reaction syndrome). Clin Rev Allergy. 1992;10(4):303-23.

11. Wang CY, Maibach HI. Immunologic contact urticaria-the human touch. Cutan Ocul Toxicol. 2013;32(2):154-60.
12. Gimenez-Arnau A. Contact urticaria and the environment. Rev Environ Health. 2014;29(3):207-15.

13. Novembre E et al. Urticaria and urticaria related skin condition/disease in children. Eur Ann Allergy Clin Immunol. 2008;40(1):5-13.

14. Davari P, Maibach HI. Contact urticaria to cosmetic and industrial dyes. Clin Exp Dermatol. 2011;36(1):1-5.

15. McFadden J. Immunologic contact urticaria. Immunol Allergy Clin N Am. 2014;34(1):157-67.

16. Zuberbier T, Maurer M. Urticaria: current opinions about etiology, diagnosis and therapy. Acta Derm Venereol. 2007;87(3):196-205.

17. Burdick AE, Mathias CG. The contact urticaria syndrome. Dermatol Clin. 1985;3(1):71-84.

18. Guin JD. The evaluation of patients with urticaria. Dermatol Clin. 1985;3(1):29-49.

19. Bourrain JL. Occupational contact urticaria. Clin Rev Allergy Immunol. 2006;30(1):39-46.

20. Kanerva L et al. Statistical data on occupational contact urticaria. Contact Dermatitis. 1996;35(4):229-33.

21. Helaskoski E, Kuuliala O, Aalto-Korte K. Occupational contact urticaria caused by cyclic acid anhydrides. Contact Dermatitis. 2009;60(4):214-21.

22. English JSC. Occupational dermatoses, in Rook's textbook of dermatology. 8th ed, In: B.J. Rook A, Champion RH, Ebling FJ, Wilkinson DS, editors. Wiley Blackwell; 2010. p. 27.1-27.21

23. Alikhan A, Chan HP, Maibach HI. Produce-induced contact urticaria and dermatitis: solanaceae and alliaceae. Contact Dermatitis. 2009;60(3):174-6.

24. Thormann H, Paulsen E. Contact urticaria to common ivy (Hedera helix cv. 'Hester') with concomitant 
immediate sensitivity to the labiate family (Lamiaceae) in a Danish gardener. Contact Dermatitis. 2008;59(3):179-80.

25. von Krogh G, Maibach HI. The contact urticaria syndrome-an updated review. J Am Acad Dermatol. 1981;5(3):328-42.

26. Greaves MW. Pathology and classification of urticaria. Immunol Allergy Clin N Am. 2014;34(1):1-9.

27. Zuberbier T. Urticaria. Allergy. 2003;58(12):1224-34.

28. Amin S, Tanglertsampan C, Maibach HI. Contact urticaria syndrome: 1997 . Am J Contact Dermat. 1997;8(1):15-9.

29. Helaskoski E et al. Prick testing with chemicals in the diagnosis of occupational contact urticaria and respiratory diseases. Contact Dermatitis. 2015;72(1):20-32.

30. Maucher OM. Anaphylactic reactions during skin tests. Hautarzt. 1972;23(3):139-40.

31. Haustein UF. Anaphylactic shock and contact urticaria after the patch test with professional allergens. Allerg Immunol. 1976;22(4):349-52.

32. Venarske D, deShazo RD. Molecular mechanisms of allergic disease. South Med J. 2003;96(11):1049-54.

33. Gonzalez-Munoz M et al. Occupational protein contact dermatitis to chicken meat studied by flow cytometry. Contact Dermatitis. 2007;57(1):62-3.

34. Foti $\mathrm{C}$ et al. Contact urticaria to raw arugula and parsley. Ann Allergy Asthma Immunol.

2011;106(5):447-8.

35. Price A et al. Oral allergy syndrome (pollen-food allergy syndrome). Dermatitis. 2015;26(2):78-88.

36. Ma S, Sicherer SH, Nowak-Wegrzyn A. A survey on the management of pollen-food allergy syndrome in allergy practices. J Allergy Clin Immunol. 2003;112(4):784-8.

37. Inomata $\mathrm{N}$ et al. Food allergy preceded by contact urticaria due to the same food: involvement of epicutaneous sensitization in food allergy. Allergol Int. 2015;64(1):73-8.

38. Asero R. Peach-induced contact urticaria is associated with lipid transfer protein sensitization. Int Arch Allergy Immunol. 2011;154(4):345-8.

39. Schroer BC, Fox CC, Hauswirth DW. Skin testing after anaphylaxis to a topical neosporin preparation. Ann Allergy Asthma Immunol. 2008;101(4):444.

40. Warshaw EM et al. North American Contact Dermatitis Group patch test results: 2009 to 2010. Dermatitis. 2013;24(2):50-9.

41. Bommarito L, Mietta S, Cadario G. Anaphylaxis after application of topical bacitracin-neomycin powder. Ann Allergy Asthma Immunol. 2015;115(1):74-5.

42. Palungwachira P. Contact urticaria syndrome and anaphylactoid reaction from topical clioquinol and bacitracin (Banocin): a case report. J Med Assoc Thail. 1991;74(1):43-6.

43. Saito $M$, Nakada T. Contact urticaria syndrome from eye drops: levofloxacin hydrate ophthalmic solution. J Dermatol. 2013;40(2):130-1.
44. Weng M et al. Life-threatening anaphylactic shock due to chlorhexidine on the central venous catheter: a case series. Int J Clin Exp Med. 2014;7(12):5930-6.

45. Krishna MT et al. Multi-centre retrospective analysis of anaphylaxis during general anaesthesia in the United Kingdom: aetiology and diagnostic performance of acute serum tryptase. Clin Exp Immunol. 2014;178(2):399-404.

46. Antunes J, Kochuyt AM, Ceuppens JL. Perioperative allergic reactions: experience in a Flemish referral centre. Allergol Immunopathol. 2014;42(4):348-54.

47. Opstrup MS et al. Standardized testing with chlorhexidine in perioperative allergy-a large single-centre evaluation. Allergy. 2014;69(10):1390-6.

48. Waton $\mathrm{J}$ et al. Contact urticaria from Emla cream. Contact Dermatitis. 2004;51(5-6):284-7.

49. Channual J, Wu JJ, Zachary CB. Localized contact urticaria caused by lidocaine/tetracaine peel. Arch Dermatol. 2009;145(4):499-500.

50. Alster TS. The lidocaine/tetracaine peel: a novel topical anesthetic for dermatologic procedures in adult patients. Dermatol Surg. 2007;33(9):1073-81.

51. Roesch A et al. Severe contact urticaria to guar gum included as gelling agent in a local anaesthetic. Contact Dermatitis. 2005;52(6):307-8.

52. Spoerl D, Scherer K, Bircher AJ. Contact urticaria with systemic symptoms due to hexylene glycol in a topical corticosteroid: case report and review of hypersensitivity to glycols. Dermatology. 2010;220(3):238-42.

53. Nutter AF. Contact urticaria to rubber. Br J Dermatol. 1979;101(5):597-8.

54. Brehler R, Sedlmayr S. Contact urticaria due to rubber chemicals? Contact Dermatitis. 1997;37(3):125-7.

55. Crippa $\mathrm{M}$ et al. Consensus document. Update on latex exposure and use of gloves in Italian health care settings. Med Lav. 2008;99(5):387-99.

56. Chen $\mathrm{Z}$ et al. Identification of hevein ( $\mathrm{Hev} b$ 6.02) in Hevea latex as a major cross-reacting allergen with avocado fruit in patients with latex allergy. J Allergy Clin Immunol. 1998;102(3):476-81.

57. Cromwell O et al. Specific IgE antibodies to platinum salts in sensitized workers. Clin Allergy. 1979;9(2):109-17.

58. Estlander $\mathrm{T}$ et al. Immediate and delayed allergy to nickel with contact urticaria, rhinitis, asthma and contact dermatitis. Clin Exp Allergy. 1993;23(4):306-10.

59. Temesvari E et al. Multicentre study of fragrance allergy in Hungary. Immediate and late type reactions. Contact Dermatitis. 2002;46(6):325-30.

60. Vanden Broecke K et al. Contact urticaria syndrome caused by direct hair dyes in a hairdresser. Contact Dermatitis. 2014;71(2):124-6.

61. Poltronieri A et al. Occupational allergic "march". Rapid evolution of contact dermatitis to ammonium persulfate into airborne contact dermatitis with rhinitis 
and asthma in a hairdresser. Med Lav.

2010;101(6):403-8.

62. Adisesh A et al. U.K. standards of care for occupational contact dermatitis and occupational contact urticaria. Br J Dermatol. 2013;168(6):116775 .

63. Nicholson PJ, Llewellyn D, English JS. Evidence-based guidelines for the prevention, identification and management of occupational contact dermatitis and urticaria. Contact Dermatitis. 2010;63(4):177-86.

64. Cirla AM. Asthma and baker's allergy: experience with health programs. G Ital Med Lav Ergon.

2011;33(1):20-5.

65. Nettis E et al. The safety of a novel sublingual rush induction phase for latex desensitization. Curr Med Res Opin. 2010;26(8):1855-9. 